\title{
Orientation and direction-of-motion response in the middle temporal visual area (MT) of NewWorld owl monkeys as revealed by intrinsic-signal optical imaging
}

\author{
Peter M. Kaskan ${ }^{1 \dagger}$, Barbara C. Dillenburger ${ }^{2}$, Haidong D. Lu ${ }^{3}$, Anna W. Roe ${ }^{1}$ and Jon H. Kaas ${ }^{1 *}$ \\ Department of Psychology, Vanderbilt University, Nashville, TN, USA \\ 2 Institute of Imaging Science, Vanderbilt University, Nashville, TN, USA \\ ${ }^{3}$ Institute of Neuroscience, Chinese Academy of Sciences, Shanghai, China
}

Edited by:

Kathleen S. Rockland, Massachusetts Institute of Technology, USA

Reviewed by:

Zoltan F. Kisvarday,

University of Debrecen, Hungary

Leah Krubitzer,

University of California, USA

*Correspondence:

Jon H. Kaas,

Department of Psychology

Vanderbilt University, 301 Wilson Hall,

11121 st Avenue South, Nashville, TN

37203, USA. e-mail: jon.h.kaas@

vanderbilt.edu

${ }^{+}$Current address:

Peter M. Kaskan, Laboratory of Neuropsychology, National Institutes of

Mental Health, 9000 Rockville Pk.,

Building 49, Rm 1B80, Bethesda, MD

20892, USA.

e-mail:kaskanpm@mail.nih.gov
Intrinsic-signal optical imaging was used to evaluate relationships of domains of neurons in middle temporal visual area (MT) selective for stimulus orientation and direction-of-motion. Maps of activation were elicited in MT of owl monkeys by gratings drifting back-and-forth, flashed stationary gratings and unidirectionally drifting fields of random dots. Drifting gratings, typically used to reveal orientation preference domains, contain a motion component that may be represented in MT. Consequently, this stimulus could activate groups of cells responsive to the motion of the grating, its orientation or a combination of both. Domains elicited from either moving or static gratings were remarkably similar, indicating that these groups of cells are responding to orientation, although they may also encode information about motion. To assess the relationship between domains defined by drifting oriented gratings and those responsive to direction-of-motion, the response to drifting fields of random dots was measured within domains defined from thresholded maps of activation elicited by the drifting gratings. The optical response elicited by drifting fields of random dots was maximal in a direction orthogonal to the map of orientation preference. Thus, neurons in domains selective for stimulus orientation are also selective for motion orthogonal to the preferred stimulus orientation.

Keywords: orientation columns, cortical modules, visual cortex, direction-of-motion columns, primates

\section{INTRODUCTION}

The middle temporal visual area (MT) is one of three of the many proposed visual areas (V1, V2 and MT) widely recognized in all primates (Kaas, 1997). MT is more myelinated and expresses more cytochrome oxidase (CO) than surrounding fields, represents the visual hemifield in a retinotopic manner, and is activated by direct projections from V1 and V2 (e.g., Allman et al., 1973; Allman and Kaas, 1974; Gattass and Gross, 1981; Tootell et al., 1985; Krubitzer and Kaas, 1990a; Rosa and Schmid, 1995; Tootell and Taylor, 1995; Kaas, 1997; Born and Bradley, 2005; Collins et al., 2005). The response properties of cells within MT have been well characterized, and the general consensus is that cells are tuned for orientation, directionof-motion, speed and/or disparity (Baker et al., 1981; Maunsell and Van Essen, 1983a,b; Albright, 1984; Albright et al., 1984; Felleman and Kaas, 1984; Tanaka et al., 1986; Lagae et al., 1993; Rosa and Elston, 1998; DeAngelis and Newsome, 1999; Born, 2000; Diogo et al., 2003). However, the spatial relationships of cells exhibiting these properties and whether there are groups of cells or modules tuned to these properties or combinations thereof, is only partially understood. Optical imaging, a technique used to study the spatial relationships of activated cell groups in the cortex is an ideal method to explore these relationships.

In optical imaging experiments in primates, drifting gratings are typically used to define orientation preference domains in V1 and V2 (Blasdel and Salama, 1986; Grinvald et al., 1986; Malach et al., 1994; Ts'o et al., 2001; Kaskan et al., 2007), in area 18 in cats (Kisvarday et al., 2001; Swindale et al., 2003) and in primate area MT (Malonek et al., 1994; Xu et al., 2004). As moving gratings contain orientation and motion, it is difficult to discern the contribution that the motion of the grating makes, if any, to orientation preference maps generated in this way. Because moving oriented gratings are typically used to map orientation preference domains in MT, orientation preference maps of MT could contain some representation of the motion of the grating. This is especially relevant since MT contains large numbers of directionally selective neurons (Zeki, 1974; 1980; Baker et al., 1981; Maunsell and Van Essen, 1983b; Albright, 1984; Albright et al., 1984; Felleman and Kaas, 1984; Lagae et al., 1993).

In the present study we compare response patterns elicited by moving gratings with those elicited by stationary flashed gratings and moving random dots to determine the spatial relationship between motion and orientation responses in MT of owl monkeys. To our knowledge, our study is the only study to demonstrate the correspondence between maps produced from static and moving oriented stimuli in MT. Our results indicate clearly that for MT, flashed, stationary gratings and moving gratings produce highly similar maps. Therefore moving gratings can be said to produce valid maps of stimulus orientation. In addition, dot fields moving perpendicular to a gratings orientation, produce direction and axisof-motion maps that overlap the orientation maps. Thus, this study 
together with previous studies suggest that neurons in owl monkey MT are sensitive to both orientation and to direction-of-movement orthogonal to their selectivity for stimulus orientation, and these neurons are grouped so that MT systematically represents both stimulus orientation and axis of movement (axis-of-movement domains consist of adjoining direction-of-movement domains preferring the same orientation).

\section{MATERIALS AND METHODS}

In general, procedures followed those used previously in our optical imaging experiments in owl monkeys (Kaskan et al., 2007). All procedures used in this study were approved by the Vanderbilt Animal Care and Use Committee and conformed to guidelines set out by the National Institutes of Health.

\section{ANIMAL PREPARATION}

The four adult owl monkeys (Aotus sp.) in this study were initially given i.m. injections of ketamine and atropine $(10 \mathrm{mg} / \mathrm{kg}$ each) and were intubated and artificially ventilated with $2 \%$ isoflurane in oxygen. Anesthetic depth was continuously monitored by EEG. Endtidal $\mathrm{CO}_{2}$, heart rate, body temperature and blood oxygen were also monitored continuously. Eyes were dilated with atropine sulfate and were refracted to focus on a computer screen, placed $28.5 \mathrm{~cm}$ from the animals' eyes. A craniotomy and durotomy were performed to expose the superior temporal sulcus (STS) and surrounding cortex. The cortex was covered with a $4 \%$ agar solution and a clear glass coverslip ( $0.15 \mathrm{~mm}$ thick) to stabilize the brain. During imaging the animal was maintained under a combination of sufentanil $(2-4 \mu \mathrm{g} /$ $\mathrm{kg} / \mathrm{hr}$ i.v.), propofol (4-8 mg/kg/hr i.v.) for anesthesia and vecuronium bromide $(0.05 \mathrm{mg} / \mathrm{kg} / \mathrm{hr}$ i.v. $)$ to maintain stability of the eyes. Values of propofol and sufentanil were adjusted within these ranges in order to keep the animal at level 2 anesthesia.

\section{OPTICAL IMAGING}

Stimuli were generated with a Visual Stimulus Generation (VSG)board (Cambridge Research Systems) and presented on a cathode ray tube (CRT) monitor (Sony Trinitron, GDM F500R) with a refresh rate of $100 \mathrm{~Hz}$. Screen extent spanned about $70^{\circ} \times 56^{\circ}$ of visual field. All stimuli were presented binocularly. The eyes were in close alignment but were not precisely aligned, as estimated from the projections of the optic disks into the visual field.

Images of reflectance change (intrinsic hemodynamic signals) corresponding to local cortical activity were acquired using Imager 3001 (Optical Imaging Inc., Germantown, NY, USA) with $630 \mathrm{~nm}$ illumination (Ramsden et al., 2001; Lu and Roe, 2008). Signal-tonoise ratio was enhanced by trial averaging (10-50 trials per stimulus condition) and by synchronization of acquisition with heart rate and respiration. Stimuli were presented for $5 \mathrm{~s}$, during which 16-20 consecutive image frames were taken (4 Hz frame rate). Inter-stimulus interval for all stimuli was $8 \mathrm{~s}$. Each frame contained $504 \times 504$ pixels (representing $8 \mathrm{~mm} \times 8 \mathrm{~mm}$ cortex area). Stimuli were presented in blocks of randomly interleaved conditions.

\section{VISUAL STIMULI}

Visual stimuli consisted of achromatic square-wave gratings and fields of random dots. Square-wave gratings ( $0.25 \mathrm{c} /$ degree $)$ reversed direction every second and moved orthogonal to the orientation of grating at $5 \mathrm{~Hz}$. Four different orientations were used $\left(0^{\circ}, 45^{\circ}\right.$, $90^{\circ}$ and $135^{\circ}$ ). The mean luminance of the gratings was $43 \mathrm{~cd} / \mathrm{m}^{2}$ and the contrast was $100 \%$. An equiluminant gray screen was used as a blank. In another instance, static gratings were flashed on and off at $1.33 \mathrm{~Hz}$. The "off" portion of this stimulus was an equiluminant gray screen. Fields of random dots $(1.5 \%)$ were moved in one of eight directions randomly interleaved at $45^{\circ}$ increments. Dots were $0.65^{\circ}$ square and drifted at $10^{\circ} / \mathrm{s}$. A field of dots (density $1.5 \%$ ) moving randomly ("wiggling") in place at $10 \%$ was used as a "blank" with motion content. We also used a cocktail "blank" (sum of all eight direction maps; cf. Bartfeld and Grinvald, 1992). Visual stimuli occupied approximately $20^{\circ}$ of the contralateral visual hemifield, while extending about $5^{\circ}$ into the ipisilateral hemifield, as estimated from the projections of the optic disks onto the visual field. Patterns of activation covering approximately $20^{\circ}$ of central MT were in agreement with previously published electrophysiological maps (Allman and Kaas, 1971). Each stimulus was presented between 20 and 40 times.

\section{COMPARISON OF MAPS ELICITED BY MOVING AND STATIONARY STIMULI}

The similarity between maps elicited by oriented gratings drifting back-and-forth and those elicited by flashed stationary gratings was assessed. From thresholded images (20\% threshold), the areas of domains in either map were measured using NIH ImageJ (Rasband, 2007) as well as the area common to each map. The percentage that domains elicited from static flashed gratings overlapped with those produced from drifting gratings was calculated (number of pixels in overlapping regions divided by number of pixels in domains from static grating maps, only domains which were fully within the field of view were included in calculation).

\section{IMAGE ANALYSIS}

All results were initially first-frame subtracted using the first two frames in each trial. We generated subtraction maps for grating stimuli (e.g., $0-90^{\circ}, 45-135^{\circ}$, etc.) and single condition blanksubtracted direction-of-motion maps from the random dot stimuli (e.g., $0^{\circ}$ - "wiggling" dots, or $45^{\circ}$ - cocktail blank, etc.). Maps were low-pass filtered with a Gaussian kernel of 3, 5 or 7 pixels, hi-pass filtered with a Gaussian kernel of 80 or 120 pixels ( 1.27 or $1.9 \mathrm{~mm}$ ) and clipped at 1 or $1.5 \mathrm{SD}$ from the mean using custom written software in Matlab. Values of signal strength $(\Delta R / R)$ taken from the region of interest (ROI) located in the representation of central vision were measured from first-frame subtracted data. To get signal strength values, the gray value of each pixel was calculated using following function: $\Delta R / R=\left(F_{5-20}-F_{1-2}\right)$, in which $\Delta R / R$ represents percentage change, $F_{5-20}$ is the average raw reflectance values of frames 5-20. $F_{1-2}$ is the average raw reflectance value of the first two frames (which thus represents baseline activity). Single-condition maps obtained in this way represent the percentage intrinsic signal change compared with baseline condition.

\section{COMPARISON OF ORIENTATION AND DIRECTION-OF-MOTION MAPS}

A ROI was defined within the representation of central vision in MT. Within this ROI, maps of activation elicited by gratings of horizontal, vertical, acute and oblique orientation were thresholded (top 20\%, bottom 20\%) using Photoshop to define horizontal 
$(\mathrm{H})$, vertical $(\mathrm{V})$, acute $(\mathrm{A})$ and oblique $(\mathrm{O})$ domains (typically considered orientation preference domains; see Introduction). Very small or unusually shaped domains (some due to blood vessel artifacts) were not included in the analysis. From the regions defined as $\mathrm{H}, \mathrm{V}, \mathrm{A}$ and $\mathrm{O}$ domains, a histogram of pixel counts was extracted from each of the corresponding eight direction preference maps obtained with drifting dots. A direction preference histogram was generated for each $\mathrm{H}, \mathrm{V}, \mathrm{A}$ and $\mathrm{O}$ domain; this procedure was also done for the complete ROI, i.e., for the collection of H, V, A and O domains. For each direction preference histogram, the numbers of pixels in the top and bottom $10 \%$ of the histogram were summed; these values were normalized by dividing each by the total number of pixels. These normalized "top + bottom" direction preference histogram values (percentage of pixels for each direction) were used to generate radial plots of pixel counts for each horizontal, vertical, acute and oblique map (see for example, Figure 5). This procedure is described in pictorial form in Figure 4.

\section{HISTOLOGY}

The animals were given a lethal dose of sodium pentobarbital $(80 \mathrm{mg} / \mathrm{kg}$ ) and when the EEG showed no electrical activity, they were perfused transcardially with phosphate buffered saline (PBS, $\mathrm{pH}$ 7.4), followed by $2 \%$ paraformaldehyde in PBS. After perfusion, the brain was removed and prepared for processing. The cortex was carefully removed from the underlying white matter, manually flattened (Krubitzer and Kaas, 1990b), held between two glass plates and stored overnight in 30\% sucrose in PBS. The cortex was cut on a freezing microtome in sections parallel to the surface at a thickness of $100 \mu \mathrm{m}$ (top three sections) and $50 \mu \mathrm{m}$ (remaining sections). Sections were processed for CO (Wong-Riley, 1979) and myelin (Gallyas, 1979). Architectonic borders apparent on sections processed for $\mathrm{CO}$ or myelin were transferred to the camera frames of interest. Photographs of the histological material were taken with a digital camera (Nikon, DXM1200F) attached to a Nikon Eclipse E800 microscope.

\section{ALIGNMENT METHODOLOGY}

Images of $\mathrm{CO}$ processed tissue or myelin were aligned to images of the surface vasculature taken in vivo with the imaging camera. Adobe Photoshop was used to align the histological sections with the optical imaging camera frames using surface vasculature and the locations of radially oriented vessels (see Kaskan et al., 2009). In some instances "stretching" was required in addition to scaling and rotating images to account for minor irregularities due to curvature of the brain in vivo, flattening the cortex and mounting the CO-processed or myelin stained tissue on slides.

\section{RESULTS}

Intrinsic-signal optical imaging was used to examine the response of the MT of owl monkeys to moving visual stimuli. Oriented gratings drifting back-and-forth orthogonal to the orientation of the grating, flashed stationary gratings or drifting fields of random dots elicited modular patterns of activation in MT. In four owl monkeys, the relative locations of these regions of activated cortex were determined. These results were related to cortical architecture in brain sections cut parallel to the surface, processed for $\mathrm{CO}$ or myelin.

\section{APPEARANCE AND LOCATION OF MT, AND ACTIVATION BY MOVING STIMULI}

MT is located on the lateral surface of the brain, just posterior to the caudal tip of the STS (Figure 1A). The size, location and extent of MT in this monkey was estimated by aligning a myelin processed section of the flattened cortex (Figure 1B) with the pattern of blood vessels on the surface of the brain (see Materials and Methods). In this case, as in other cases, staining within MT was fairly homogenous, and there was no evidence of variations in myelin density that would suggest compartments, as sometimes reported (Van Essen et al., 1981; Tootell et al., 1985; Krubitzer and Kaas, 1990b; Kaas and Morel, 1993; Rosa and Elston, 1998). This could be due to the extensive nature of our imaging procedures, which may have interfered with subtleties in myelination or enzyme activity (CO). The box surrounding MT in Figure 1A indicates the region of cortex imaged in Figures 1B-D. MT was activated by gratings drifting bidirectionally (Figure 1C, subtraction map of horizontal minus vertical) and by a drifting field of random dots (Figure 1D, single upward drifting direction). As the two types of stimuli occupied the same proportion of the contralateral visual hemifield, the extent of cortex activated by the gratings and the random dots was comparable. The maximum percent signal change due to the drifting gratings and drifting fields of random dots, measured across all pixels within approximately the central $20^{\circ}$ of central vision (see boxed region, Figures 1C,D), was -0.066 and -0.082 , respectively. There were no differences in signal strength between different orientations of drifting gratings or between different directions of drifting random dots. Thus, consistent with previous studies, MT can be reliably activated by gratings drifting back-and-forth, and by drifting fields of random dots.

The extent of cortex activated by drifting gratings and drifting random dots was smaller than the region which appeared darkly stained in a myelin processed section (Figure 1B). It is possible that this myelin dense region included MST, or portions of MST (cf. Kaas and Morel, 1993), which were not strongly activated by our stimulus conditions. Another explanation for this discrepancy could be that the extent of visual field stimulated (about $70^{\circ} \times 56^{\circ}$ ) is smaller than the entire visual field represented in MT. Nevertheless, the extent of the activation elicited by the gratings and the random dots corresponded to the posterior extent of the densely stained region apparent in the myelin stain (Figure 1B).

To help visualize the sizes and shapes of MT domains elicited by drifting gratings and drifting fields of random dots, and their relationship to one another, a portion of MT representing about $20^{\circ}$ of central vision was selected for further analysis (see boxed region, Figures 1C,D). Thresholding procedures (see Materials and Methods) were used to identify regions of MT within the rectangle that responded to drifting gratings oriented $0^{\circ}$ (horizontal, black regions in Figure 1E) and $90^{\circ}$ (vertical, white regions in Figure 1E). The same thresholding procedures were used to identify regions of MT within the rectangle which were activated by random dots moving up (direction of $0^{\circ}$ ); these regions are outlined in white and are shown with respect to horizontal and vertical domains in Figure 1E. The up-direction domains, roughly circular in appearance, predominantly overlapped portions of horizontal domains (black) and not vertical domains (white), as well as some cortex that was activated below our thresholding level. In fact, none of the updirection domains encircled the vertical gratings domains, which 

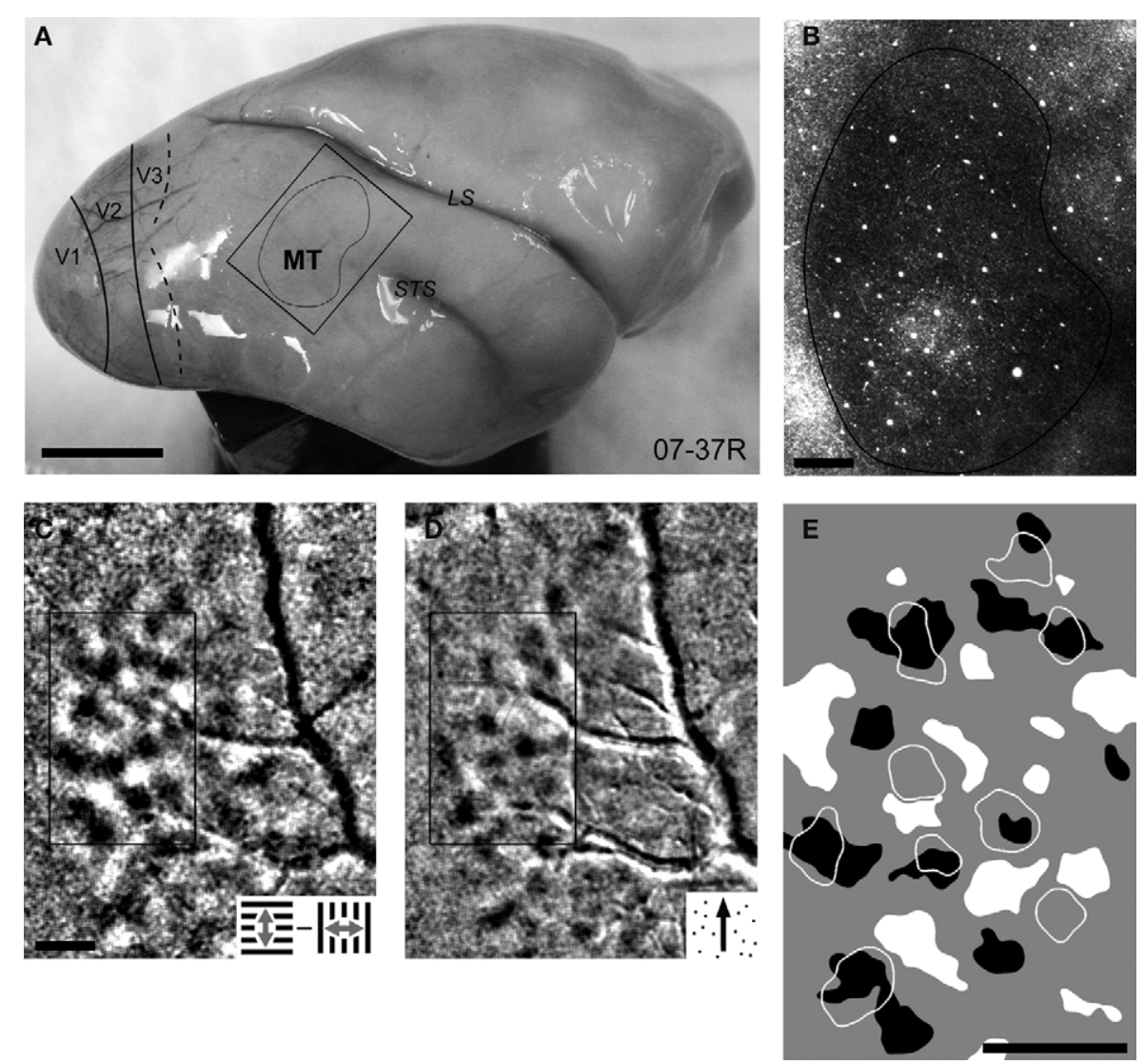

FIGURE 1 |The activation of MT by drifting gratings and random dots in case 07-37R. (A)The location of MT and other visual areas on a lateral-surface view of the brain. The lateral sulcus (LS) and superior temporal sulcus (STS) are indicated. The box surrounding MT outlines the field of view presented in (B-D). (B) A section of flattened cortex stained for myelin revealed the borders of MT (indicated in A). (C) A subtraction map of horizontal-vertical gratings and (D) a map of direction preference (up - cocktail blank). Both (C,D) were clipped at 1.5 SD. The region of interest (ROI) taken from the representation of central vision in MT is indicated with a thin black line in (C,D). This region represents about $20^{\circ}$. (E) The thresholded (see Materials and Methods) optical imaging maps from the $\mathrm{ROI}$ in (C,D). Black regions indicate horizontal "orientation" domains, white regions indicate vertical "orientation" domains. The regions outlined in white indicate the up-direction domains. Note that the up domains enclose only portions of horizontal (black) domains, which moved up and down. Scale bar in (A): $5 \mathrm{~mm}$. Scale bar in (B-E): $1 \mathrm{~mm}$. were activated by the vertical grating moving horizontally left and right (white domains, Figure 1E). As parts of the domains sensitive to up and down movements of horizontal gratings are activated by up movements of random dots, other parts of those domains may be sensitive to down movements of random dots. We also note that horizontal domains often appeared larger and more elongated in shape horizontal (black) domains. This led us to the hypothesis that horizontal orientation domains in MT may coincide with pairs of up and down direction-of-motion domains.

To test the significance of the motion of the grating in producing orientation preference maps, the responses elicited from stationary flashed gratings were compared to those from moving gratings. This was conducted in two hemispheres of one monkey. In Figure 2, two horizontal-vertical subtraction maps are shown from a portion of MT representing approximately the central $20^{\circ}$ of the upper field. The contour plots overlaid upon each image were generated from a thresholded image of the underlying orientation preference map. The map in Figure 2A, produced from gratings moving back-andforth is remarkably similar to the map shown in Figure 2B, which was elicited by static, flashed gratings. Figure $2 \mathrm{C}$ demonstrates the overlap between the two maps, which was 77\% (pixel number within overlapping zones divided by all pixels in domains from static grating map). In the three other cases analyzed, gratings drifting back-andforth were used to generate orientation preference maps.

In a third monkey (case 10-110), moving gratings and random dots were used to activate MT. A photograph of the surface of the brain indicated the blood vessel pattern in the region of MT (Figure 3A). A superficial section of flattened cortex from this region was stained for CO (Figure 3B) to reveal the surface vasculature, which was used to align the optical imaging maps to a myelin stained section from the flattened cortex (Figure 3C). The myelin dense region corresponds to $\mathrm{MT}$. The large black square in Figure 3C indicates the camera frame shown in Figure 3A, corresponding to the extent of the $\mathrm{CO}$-stained section of the flattened cortex shown in Figure 3B. The region indicated by a small black square in Figure 3C, representing approximately $30^{\circ}$ of central and paracentral vision of the upper quadrant of MT, represents the portion of MT from which orientation maps (Figure 3D) and direction-of-motion maps (Figure 3E) were taken.

As in the first case, drifting dots activated MT more strongly than drifting gratings. The maximum percent signal change due to the drifting gratings and the drifting fields of random dots measured 

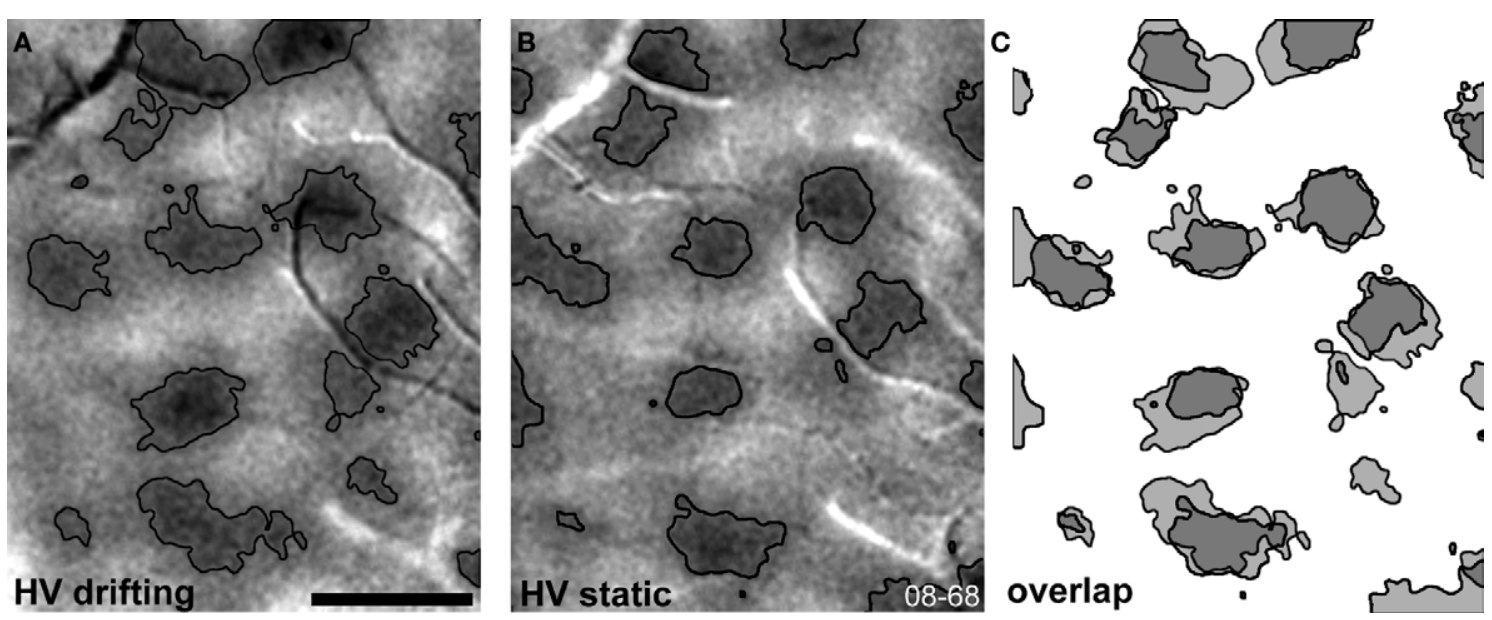

FIGURE 2 | Comparison of maps elicited by moving and stationary (flashed) gratings, case 08-68L. (A) A horizontal-vertical subtraction map is shown, produced from gratings drifting back-and-forth. Overlaid upon this map is a contour plot produced from a thresholded horizontal-vertical subtraction map. (B) A horizontal-vertical subtraction map produced from static gratings flashed at $1.33 \mathrm{~Hz}$, with corresponding contour plot. The overlap between the two thresholded maps is shown in (C). Clipping: 1 SD. Scale bar: $1 \mathrm{~mm}$ from within the sampled region was -0.090 and -0.270 , respectively. There were no differences in signal strength for different orientations of drifting gratings or different directions of drifting random dots.

Differences in the sizes of domains activated by gratings moving back-and-forth and dots moving in one direction can be seen by comparing panels in Figure 3D with those in Figure 3E. A subtraction map of horizontal-vertical grating activation (Figure 3D, top) can also be compared with a subtraction map of acute-oblique gratings (AO, Figure 3D, bottom). Note that the dark (vertical orientation) and light (horizontal orientation) regions were similar in size and shape to the dark (acute orientation) and light (oblique orientation) domains, but different in location, as expected for an orderly representation of stimulus orientation. These results are similar to the eight direction-of-motion maps evoked by moving dots (Figure 3E); the direction-of-motion domains were similar in size for each direction, and varied systematically in location from one direction to the next. Colored outlines of motion domains within a local region of MT illustrates that the direction domains shift in an orderly pinwheel-like fashion within a local region. As in the first cases, regions of cortex activated by bidirectional drifting gratings (Figure 3D) were slightly larger and more elongated than those activated by unidirectional drifting fields of random dots (Figure 3E).

The location of MT in our fourth case, 07-46R was similar to the cases illustrated in Figures 1 and 3. From the portion of MT in case $07-46 \mathrm{R}$, representing approximately $20^{\circ}$ to $30^{\circ}$ of central vision (see Figures $4 \mathrm{C}, \mathbf{F}$ ), the maximum percent signal change due to the drifting gratings and the drifting fields of random dots was -0.195 and -0.270 respectively.

\section{RELATIONSHIP BETWEEN ORIENTATION PREFERENCE DOMAINS AND DIRECTION-OF-MOTION DOMAINS IN MT}

Domains activated by gratings moving back-and-forth are somewhat larger and more elongated than those activated by drifting dots. Thresholded images of the representation of $20-30^{\circ}$ of central vision of MT in three cases (see Materials and Methods) are shown in Figure 4. Horizontal (black) and vertical (white) orientation domains (top row) can be compared with acute (black) and oblique (white) domains (bottom row) as well as with domains activated by fields of random dots (thin gray lines) drifting upward $\left(0^{\circ}\right.$, top row) and left to right at $45^{\circ}$ (bottom row). The horizontal and vertical domains (top row), and acute and oblique domains (bottom row) were larger ( $t$-test, $p=0.011$ ) and more elongated ( $t$-test, $p=0.00001$ ) than the direction preference domains (see Table 1).

A quantitative method to assess the correspondence between domains elicited by moving gratings and drifting fields of random dots was developed (Figure 5). An optical imaging subtraction map of horizontal-vertical gratings (Figure 5A) was thresholded for horizontal (black) and vertical (white) domains (Figure 5B). For comparison, the horizontal domains are shown in light gray atop a map of $45^{\circ}$ direction preference (Figure 5C). This same horizontal domain mask is then shown atop each of the eight direction preference maps (Figure 5D). A histogram of grayscale values from each of the masked portions of the eight direction preference maps was used to create a radial plot of the proportional overlap of the thresholded domains for the up and down movements of the horizontal grating with each of the eight direction-of-motion domains (Figure 5E). This radial plot shows that within horizontal orientation domains, the strongest direction response was to drifting dots in the vertical direction. Weaker responses occurred for upward movements at $315^{\circ}$ and $45^{\circ}$. Still weaker responses in the overlap zone occurred for movements in the horizontal and downward directions. Thus, the up and down motion of the horizontal gratings in this case most strongly activated portions of MT selectively activated by upward moving random dots. Surprisingly, there was little overlap with portions of MT activated by downward moving random dots (but see Discussion).

In Figure 6, "direction tuning curves" depict the relations of domains activated by the back-and-forth motion of the gratings of each orientation, to direction-of-motion domains. In almost every 

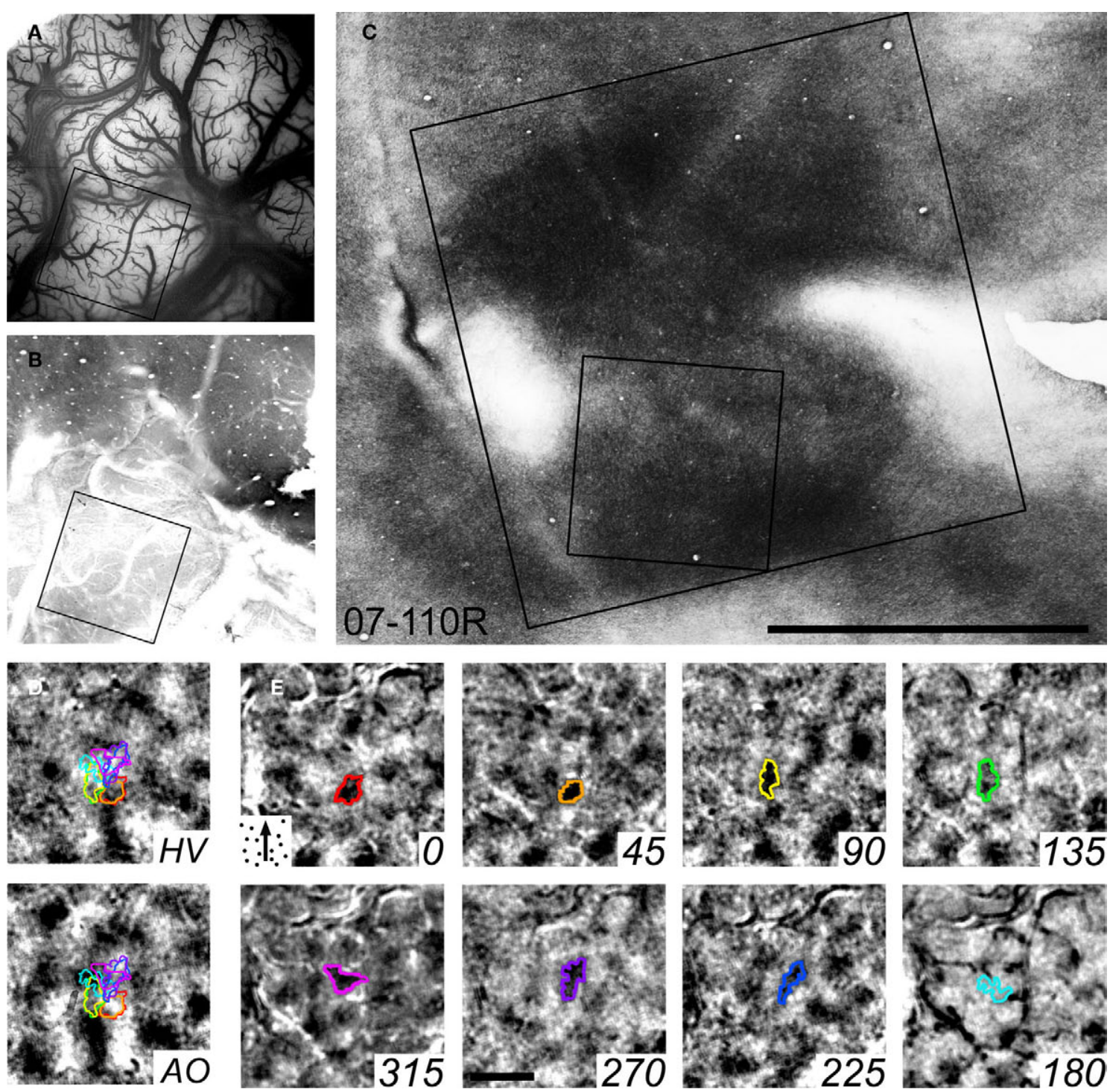

FIGURE 3 |The activation of MT by drifting gratings and random dots in case 07-110R. (A) A photograph of the surface of the brain, taken in vivo. (B) The corresponding section of the flattened cortex stained with cytochrome oxidase (CO) preserves the surface blood vessel pattern. The field of view of $(\mathbf{A}, \mathbf{B})$ is $8 \mathrm{~mm} \times 8 \mathrm{~mm}$. (C) A section of the flattened cortex stained with myelin to reveal the borders of MT. White regions correspond to superficial cortical layers that stain weakly for myelin, as the sectioned cortex was not completely flat to the superior temporal sulcus on the right and a dimple along a blood vessel on the left. The large square in (C) is the imaging camera frame (field of view in $\mathbf{A}, \mathbf{B})$ and the small square represents the cortical region further analyzed, corresponding to approximately $30^{\circ}$ of vision of the upper hemifield, shown in (D,E). (D) Orientation maps. A subtraction map of horizontal-vertical gratings $(\mathrm{HV})$ and acute-oblique gratings (AO) is shown. (E) Direction maps. Eight direction-of-motion maps are shown, representing directions of moving dots (minus cocktail blank, see Materials and Methods) for the angles 0-315 ${ }^{\circ}$ Clipping: 1.5 SD. Scale bar in (C): $5 \mathrm{~mm}$. Scale bar in (D,E): $1 \mathrm{~mm}$. tuning curve, the maximal "direction component" in response to unidirectional drifting random dots was aligned with (or close to alignment with) the axis of motion of the moving grating.

In cases 07-37 and 07-46, the direction response for portions of MT best activated by up and down movements of the horizontal grating was greatest for the upward movement, but in case $07-110 \mathrm{a}$ nearly comparable response for the upward and downward movements is apparent. Consistent with this, horizontal movements of dots within regions of MT best activated by gratings moving up and down consistently produced poor responses. Likewise, movements of an oblique grating from $135^{\circ}$ to $315^{\circ}$ produced the strongest response for cases $07-37$ and $07-46$, while case $07-110$ gave a similar, although less robust response to these directions. The relationship of one or the other or both of the direction-ofmotion of the oriented gratings to dot motion direction was not as marked for cases $07-37$ and 07-46 for the horizontal motion domains of the vertical grating, but a strong preference for the $270^{\circ}$ to $90^{\circ}$ direction of horizontal movement was apparent for case 07-110. Finally, the activation of domains for directions of moving dots that overlapped those for the back-and-forth motion 

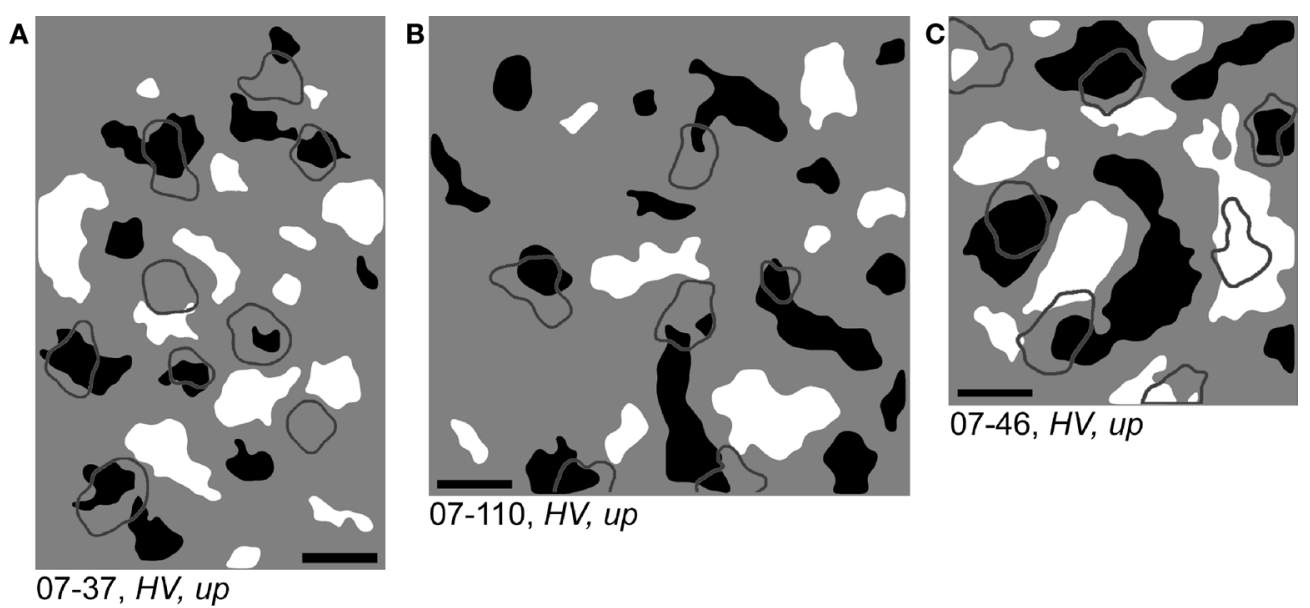

07-110, HV, up

07-46, HV, up
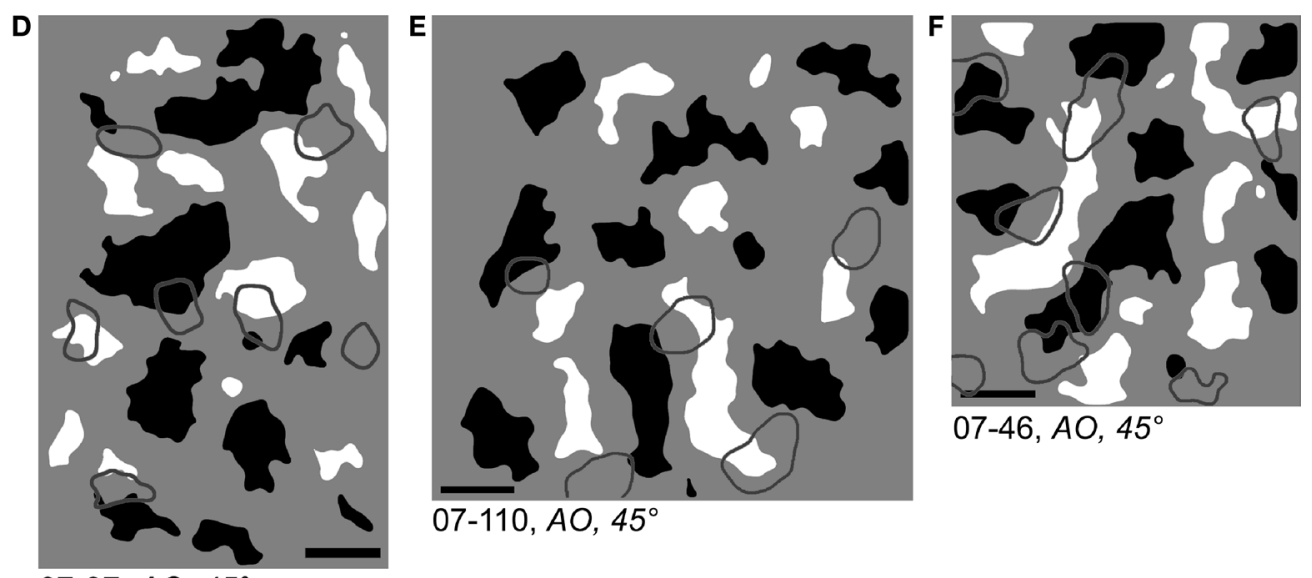

$07-46, A O, 45^{\circ}$

$07-110, A O, 45^{\circ}$

$07-37, A O, 45^{\circ}$

FIGURE 4 |The appearance of axis-of-motion and direction-of-motion domains in MT in three cases. The locations of thresholded regions of activation elicited by drifting oriented gratings and drifting fields of random dots are shown with respect to one another. Thresholded horizontal-vertical maps are shown in (A-C), whereas thresholded acute-oblique maps are shown in (D-F). Horizontal domains $(H)$, top row and acute domains $(A)$, bottom row are indicated in black. Vertical domains $(\mathrm{V})$, top row and oblique domains $(\mathrm{O})$ bottom row are indicated in white. Up domains (top row) and $45^{\circ}$ domains (bottom row) are indicated with gray lines. Black and white domains (axis-of-motion) domains appear larger and more elongated than direction-of motion domains (circled in gray). Scale bar: $500 \mu \mathrm{m}$. of the acute grating moving obliquely also varied across cases, with the upward movements of dots most strongly activated by the overlap region in cases 07-37 and 07-46, while both upward and downward moving dots activated the overlap regions for case 07-110. Together, all results indicate that there is a relationship between the direction-of-movement domains and the orientation domains, and that at least one, and sometimes two of the directions of movement of random dots activated domains that correlated with the axis of movement of the gratings. From this data, we conclude that MT contains both maps of orientation preference and direction-of-motion preference and that orientation and direction preference maps are orthogonal to one another and arranged in an orderly manner.

\section{DISCUSSION}

In MT, domains defined by drifting oriented gratings have been referred to as orientation preference domains (Malonek et al., 1994; Xu et al., 2004). But because moving gratings contain orientation and motion components, the interpretation of maps of activation evoked by such stimuli is complicated, especially in MT, where most cells are sensitive to the direction of a moving stimulus. Our first goal was to determine the degree to which patterns of activation evoked by moving oriented stimuli are similar to those evoked by stationary oriented stimuli. We found that they are in fact highly similar, with large degree of overlap (77\%) between the maps. Both methods provide similar maps of orientation preference in MT. We then sought to determine how orientation preference domains are related to direction-of-motion domains defined by stimuli lacking a prominent orientation component, such as drifting fields of random dots. Results indicate that MT contains maps of orientation preference and directionof-motion preference, and that these maps are inextricably linked. These findings are consistent with previous studies suggesting that "orientation" and "motion" domains may be viewed as spatiotemporal energy domains (cf. Adelson and Bergen, 1985; Basole et al., 2003). However, this does not exclude the view that cortex may be composed of multiple interleaved or interdigitated maps (e.g., Swindale, 2000; Swindale et al., 2000). Indeed, the degree to which 
Table 1 | Size comparison of orientation (axis of motion) domains and direction domains.

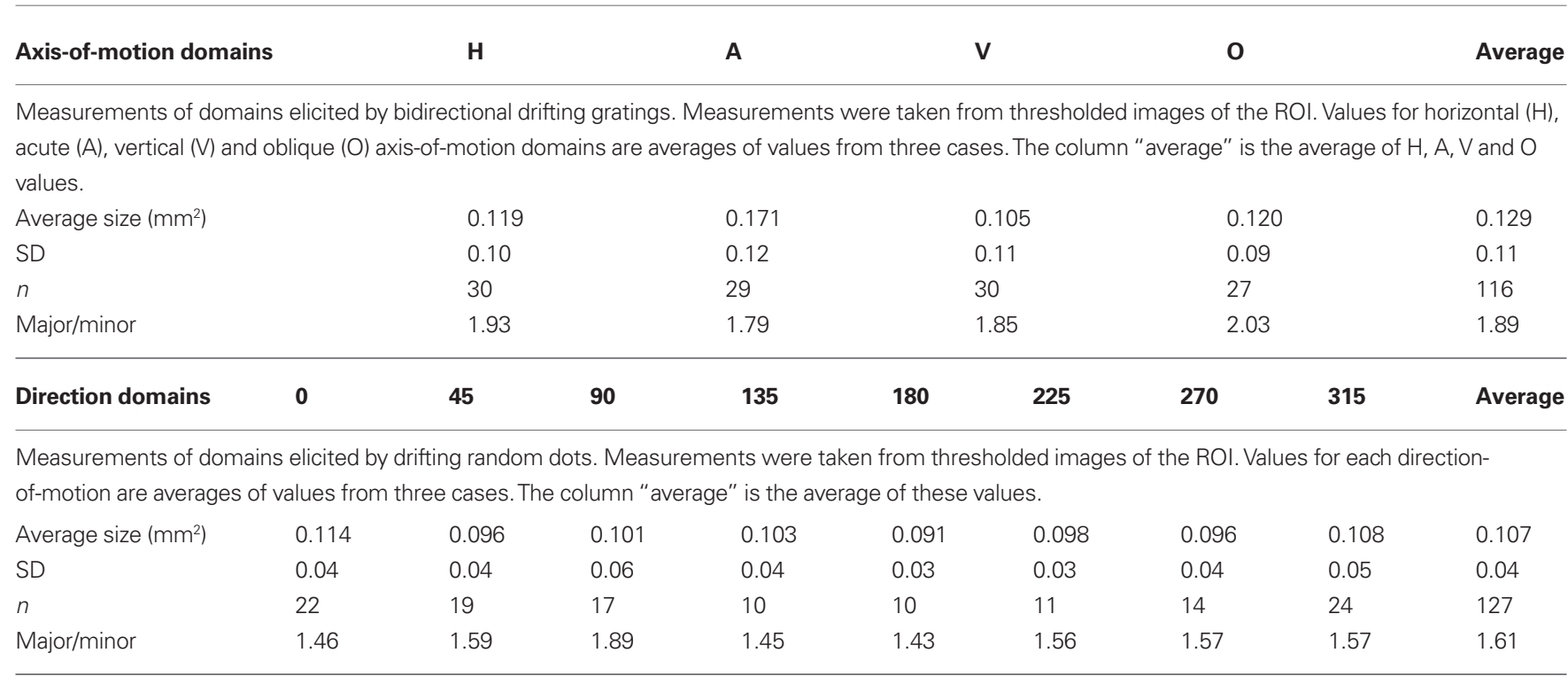

The size of domains elicited by drifting gratings was significantly larger (average $=0.129 \mathrm{~mm}^{2}$ ) than for drifting random dots (average $0.107 \mathrm{~mm} \mathrm{~m}^{2}$ ( $(\mathrm{t}$-test, $p=0.011$ ). When an ellipse was fit to each domain, and the lengths and ratios of major and minor axes of the fit ellipse measured, domains elicited by drifting gratings were significantly more elongated (major/minor axis, 1.89) than those elicited by drifting random dots (major/minor axis, 1.61) (t-test, $p=0.00001)$.

a spatiotemporal energy map may be divided into separable maps may depend on the extremes to which one parameter dominates responses at any one location.

\section{ACTIVATION OF MT BY MOVING STIMULI}

In the present experiments, gratings drifting back-and-forth, stationary flashed gratings and drifting fields of random dots elicited patchy patterns of activation that corresponded to the size, shape and location of MT as defined with stains for myelin, as previously demonstrated (Malonek et al., 1994; Xu et al., 2004; Collins et al., 2005). Drifting gratings, stationary flashed gratings and drifting fields of random dots activated MT in a manner parsimonious with the size and extent of visual field stimulation. In addition, we found an orderly organization of orientation preference and direction-of-motion preference.

Although drifting gratings and drifting fields of random dots activated a similar extent of MT, the drifting random dots produced a stronger signal change than the drifting gratings in each case. It is possible that the drifting oriented gratings (orientation map) had some inhibitory effect on the responses of some cells in MT. Inhibitory surrounds of MT cells, as previously characterized (Allman et al., 1985; Tanaka et al., 1986; Born and Tootell, 1992; Raiguel et al., 1995; Xiao et al., 1997; ), could lead to a diminished population response in the strength of the optical signal relative to that elicited from random dot fields. Another explanation is that grating movement in one direction followed by movement in the opposite direction activates and then extinguishes the responses of cells tuned to a given direction-of-motion, creating a weaker net signal change. Alternatively, drifting oriented gratings may activate only a sub-population of directionally tuned neurons, those that are tuned to orientation perpendicular to direction-of-movement; some MT neurons are maximally responsive to an orientation that is not perpendicular to its direction-of-movement (cf. Maunsell and Van Essen, 1983a,b). Thus, while we find that domains activated by the gratings were on average larger than those by the moving random dots, they are not double in size due perhaps to a number of factors, including differences in suppressive influences between these stimuli.

\section{RELATIONSHIP BETWEEN ORIENTATION PREFERENCE AND DIRECTION- OF-MOTION PREFERENCE}

One goal of the present study was to determine how patterns of activation in MT evoked by gratings drifting back-and-forth or stationary flashed gratings are related to domains defined by unidirectionally drifting fields of random dots. Previous optical imaging studies of V1 and V2 in primates (Blasdel and Salama, 1986; Grinvald et al., 1986; Malach et al., 1994; Ts'o et al., 2001; Kaskan et al., 2007) and carnivores (Shmuel and Grinvald, 1996; Weliky et al., 1996) have used drifting gratings at different angles to identify groups of cells responsive to a particular orientation. In V1 and V2 of primates, where many cells in the superficial layers of the cortex are responsive to the orientation of a stimulus, activated regions of cortex are best characterized as orientation preference domains, and contain cells tuned to a particular orientation (Grinvald et al., 1986; Maldonado et al., 1997; Ohki et al., 2006). In addition, cells in V1 and V2 of primates show similar orientation tuning curves to stationary or drifting gratings (Burkhalter and Van Essen, 1986 ; Muller et al., 2001). Therefore, optically imaged domains in $\mathrm{V} 1$ and $\mathrm{V} 2$ produced in response to drifting oriented gratings are best interpreted as orientation preference domains. Yet, to date, to our knowledge, optical imaging maps of orientation preference elicited from stationary gratings in V1 or V2 in any species have not been published. Furthermore, there are no studies directly comparing domains obtained from moving and stationary 

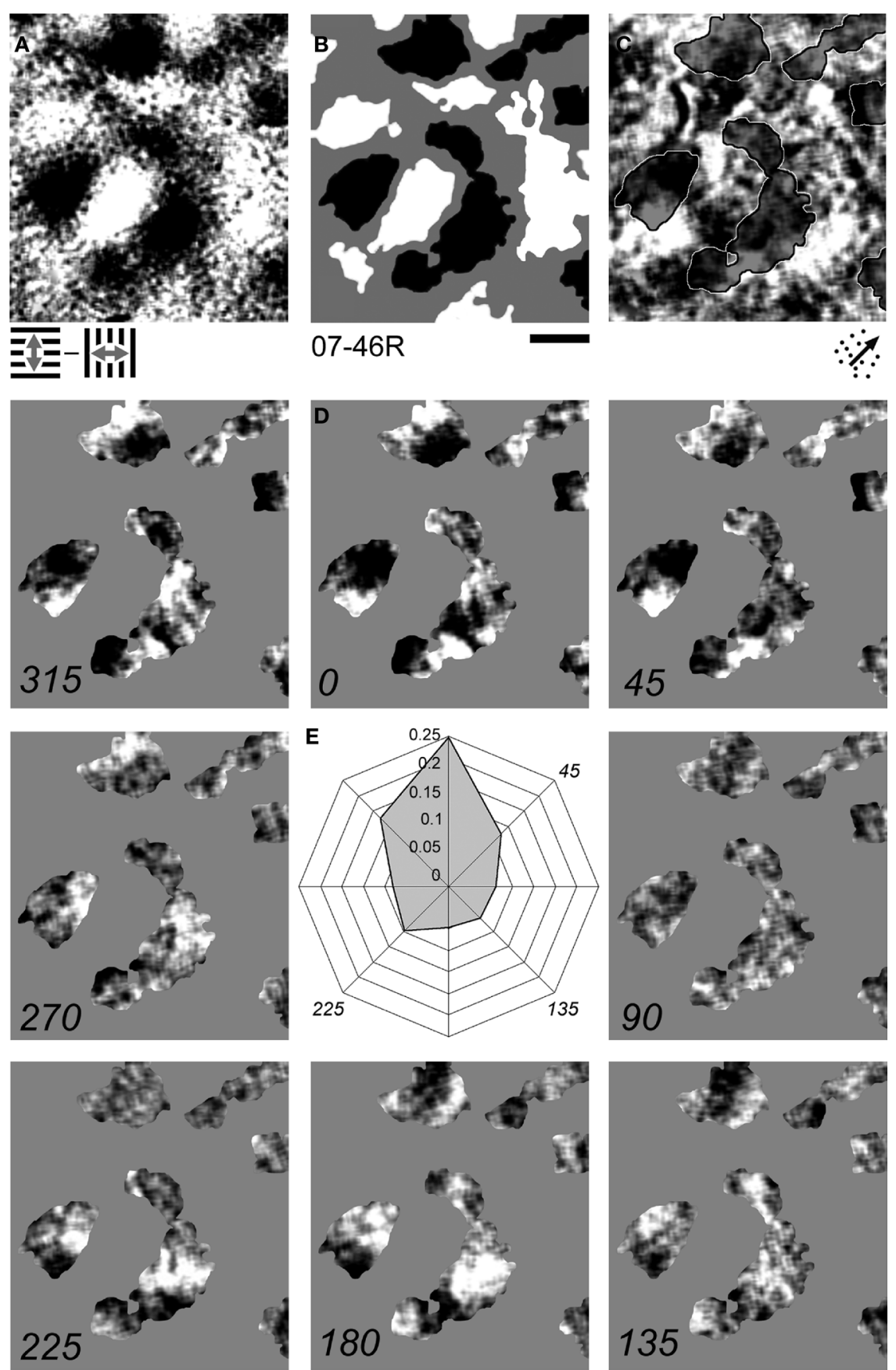

FIGURE 5 | Methodology to reveal the relationship between axis-of-motion and direction-of-motion domains. The procedure illustrated here produces a radial plot showing the direction-of-motion response for all horizontal $(\mathrm{H})$ domains within the ROI (see Materials and Methods). In (A), a HV map is shown. In (B), the same map has been thresholded (see Materials and Methods). The horizontal domains defined through thresholding have been overlaid upon a map of direction preference $\left(45^{\circ}\right)$ in (C). In (D), this "horizontal mask" has been overlaid upon each direction preference map, revealing the direction preference within the horizontal domains. For each of the maps shown in (D) a pixel histogram (number of pixels for each grayscale value) was generated. The top and bottom $10 \%$ of each histogram was summed, normalized and plotted in radial manner, shown in (E). Scale bar: $500 \mu \mathrm{m}$. gratings (cf. Malonek et al., 1994). We therefore do not know the degree to which motion is involved in the organization of orientation preference maps generated in this manner. The present study addresses this ambiguity in MT, an area containing many neurons tuned to direction-of-motion.
In MT, the majority of cells encode direction-of-motion (Zeki, 1974, 1980; Baker et al., 1981; Maunsell and Van Essen, 1983a,b; Albright, 1984; Felleman and Kaas, 1984) and few show responses to static or flashed oriented stimuli (Baker et al., 1981; Maunsell and Van Essen, 1983a,b). In an optical imaging study of MT, Malonek 


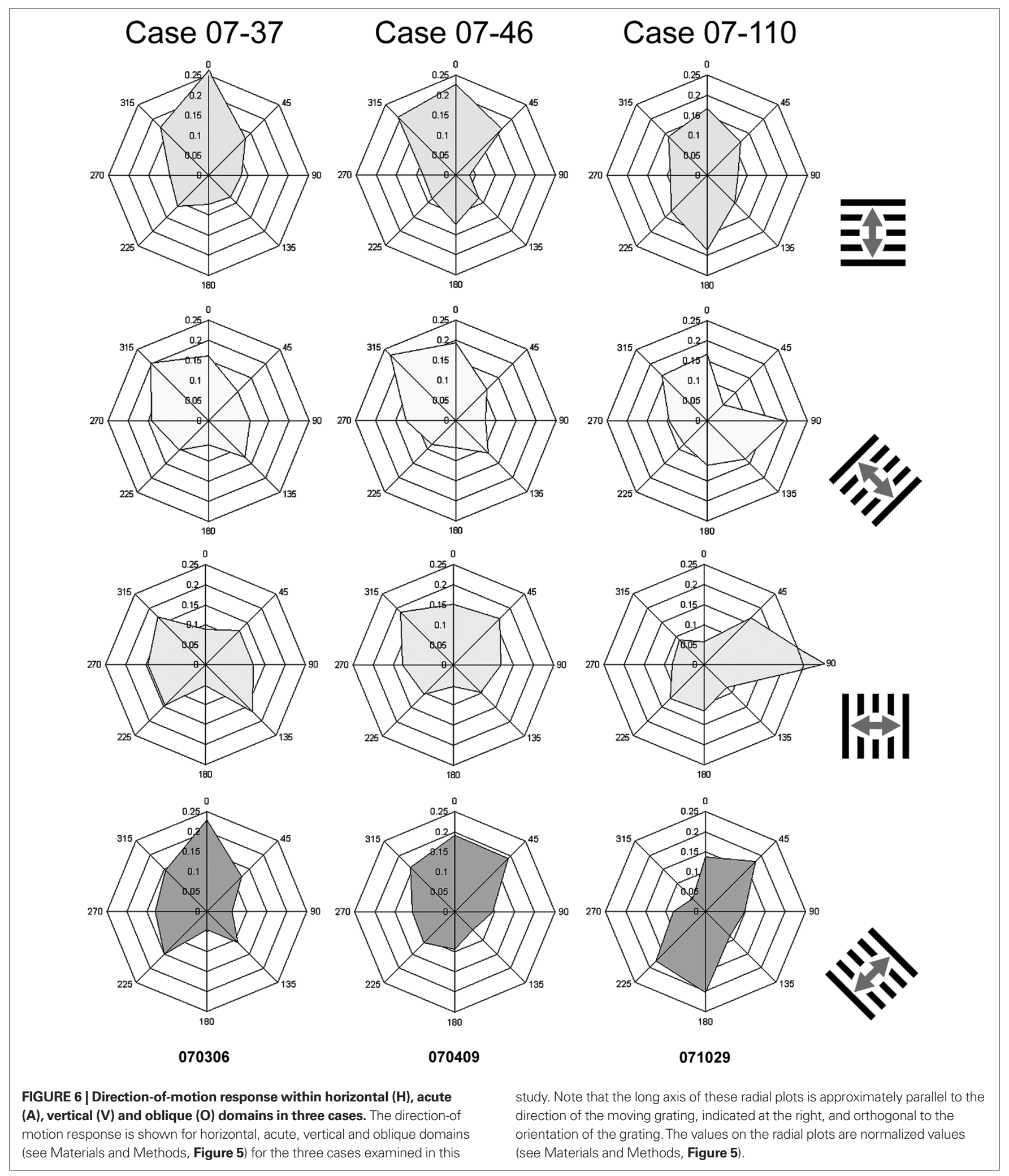

et al. (1994) commented that oriented static gratings produced weak responses, three times weaker than the equivalent moving grating. In prosimian galagos, Xu et al. (2004) used drifting oriented gratings to define orientation columns, and used drifting fields of random dots to define direction-of-motion columns. Although each of these studies utilized drifting gratings to define orientation columns, the authors concluded that the representation of orientation preference was orthogonal to direction preference. As the orientation maps 
were produced with moving gratings, it was unclear if direction of orientation or direction-of-motion characterized the "orientation" domains. Our results indicate that maps produced from gratings drifting back-and-forth can indeed be regarded as orientation preference maps, something that, until now, has not been adequately demonstrated in MT, or in any other visual cortical area. For example, the high degree of similarity between patterns of activation evoked by moving oriented stimuli and stationary flashed oriented stimuli is apparent in Figure 2. We found that both of these stimuli evoke nearly the same orientation preference map in MT. It would be interesting to determine if orientation preference maps produced in either of these ways are similar in other cortical areas, in other species.

Our interpretation of the present results is in accord with imaging data produced by moving gratings by Malonek et al. (1994) and Xu et al. (2004), and suggests that MT is composed of patches of cells responsive to specific orientations and orthogonal directionsof-motion. The results of the present experiments suggest that MT is modularly organized for the representation of stimulus orientation and direction-of-motion, and that these maps are orthogonal to one another, indicating the presence of orderly arranged groups of cells or modules involved in encoding both orientation and direction-of-motion.

Modules of the visual system, like those we describe in MT, may display a combination of architectonic, functional and connectional details that render one particular location within a cortical area different from adjacent locations. The primate visual system, perhaps the most well understood cortical sensory system is large relative to that of other mammals and has a number of well-defined cortical areas. As such, it is well suited for examining the relationship between function and structure. Results reported herein add to our understanding of the neocortex as a structure that has evolved the ability to simultaneously represent, in modular fashion, multiple stimulus features within the confines of a two-dimensional map of the sensory periphery, such as that found in MT.

The term "module" is widely used in the neuroanatomical literature, in the study of brain function and in the cognitive sciences. Though this term is in common use in a wide variety of disciplines, a clear understanding of how anatomical and functional modularity might be related has yet to be reached (cf. Kaas, 1990; Purves et al., 1992; Lund et al., 1993; Malach et al., 1994; Mountcastle, 1997; Manger et al., 1998; Kim et al., 1999; Swindale, 2000; Andersen and Buneo, 2002; Diamond et al., 2003; Chklovskii and Koulakov, 2004; Graziano and Aflalo, 2007; Op de Beeck et al., 2008), yet we believe a good starting point is an exploration of the neocortex using a combination of functional and anatomical methods.

\section{ACKNOWLEDGMENTS}

This work was supported by National Eye Institute Grants EY002686 (to Jon H. Kaas), EY11744 and DA023002 (to Anna W. Roe) and T32 EY007135 (to Peter M. Kaskan).

\section{REFERENCES}

Adelson, E. H., and Bergen, J. R. (1985). Spatiotemporal energy models for the perception of motion. J. Opt. Soc. Am. A 2, 284-299.

Albright, T. D. (1984). Direction and orientation selectivity of neurons in visual area MT of the macaque. $J$. Neurophysiol. 52, 1106-1130.

Albright, T. D., Desimone, R., and Gross, C. G. (1984). Columnar organization of directionally selective cells in visual area MT of the macaque. J. Neurophysiol. 51, 16-31.

Allman, J., Miezin, F., and McGuinness, E. (1985). Stimulus specific responses from beyond the classical receptive field: neurophysiological mechanisms for local-global comparisons in visual neurons. Annu. Rev. Neurosci. 8 , 407-430.

Allman, J. M., and Kaas, J. H. (1971). A representation of the visual field in the caudal third of the middle temporal gyrus of the owl monkey (Aotus trivirgatus). Brain Res. 31, 85-105.

Allman, J. M., and Kaas, J. H. (1974). The organization of the second visual area (V II) in the owl monkey: a second order transformation of the visual hemifield. Brain Res. 76, 247-265.

Allman, J. M., Kaas, J. H., and Lane, R. H. (1973). The middle temporal visual area (MT) in the bushbaby, Galago senegalensis. Brain Res. 57, 197-202.
Andersen, R. A., and Buneo, C. A. (2002). Intentional maps in posterior parietal cortex. Annu. Rev. Neurosci. 25, 189-220.

Baker, J. F., Petersen, S. E., Newsome, W. T., and Allman, J. M. (1981). Visual response properties of neurons in four extrastriate visual areas of the owl monkey (Aotus trivirgatus): a quantitative comparison of medial, dorsomedial, dorsolateral, and middle temporal areas. J. Neurophysiol. 45, 397-416.

Bartfeld, E., and Grinvald, A. (1992). Relationships between orientationpreference pinwheels, cytochrome oxidase blobs, and ocular-dominance columns in primate striate cortex. Proc. Natl. Acad. Sci. U.S.A. 89, 11905-11909.

Basole, A., White, L. E., and Fitzpatrick, D. (2003). Mapping multiple features in the population response of visual cortex. Nature 424, 986-990.

Blasdel, G. G., and Salama, G. (1986). Voltage-sensitive dyes reveal a modular organization in monkey striate cortex. Nature 321, 579-585.

Born, R. T. (2000). Center-surround interactions in the middle temporal visual area of the owl monkey. J. Neurophysiol. 84, 2658-2669.

Born, R. T., and Bradley, D. C. (2005). Structure and function of visual area MT. Annu. Rev. Neurosci. 28, 157-189.
Born, R. T., and Tootell, R. B. (1992). Segregation of global and local motion processing in primate middle temporal visual area. Nature 357, 497-499.

Burkhalter, A., and Van Essen, D. C. (1986). Processing of color, form and disparity information in visual areas VP and V2 of ventral extrastriate cortex in the macaque monkey. $J$. Neurosci. 6, 2327-2351.

Chklovskii, D. B., and Koulakov, A. A. (2004). Maps in the brain: what can we learn from them? Annu. Rev. Neurosci. 27, 369-392.

Collins, C. E., Xu, X., Khaytin, I., Kaskan, P. M., Casagrande, V. A., and Kaas, J. H. (2005). Optical imaging of visually evoked responses in the middle temporal area after deactivation of primary visual cortex in adult primates. Proc. Natl. Acad. Sci. U.S.A. 102, 5594-5599.

DeAngelis, G. C., and Newsome, W. T. (1999). Organization of disparityselective neurons in macaque area MT. J. Neurosci. 19, 1398-1415.

Diamond, M. E., Petersen, R. S., Harris, J. A., and Panzeri, S. (2003). Investigations into the organization of information in sensory cortex. J. Physiol. Paris 97, 529-536.

Diogo, A. C., Soares, J. G., Koulakov, A., Albright, T. D., and Gattass, R. (2003). Electrophysiological imaging of functional architecture in the cortical middle temporal visual area of Cebus apella monkey. J. Neurosci. 23, 3881-3898.

Felleman, D. J., and Kaas, J. H. (1984). Receptive-field properties of neurons in middle temporal visual area (MT) of owl monkeys. J. Neurophysiol. 52, 488-513.

Gallyas, F. (1979). Silver staining of myelin by means of physical development. Neurol. Res. 1, 203-209.

Gattass, R., and Gross, C. G. (1981).Visual topography of striate projection zone (MT) in posterior superior temporal sulcus of the macaque. J. Neurophysiol. 46, 621-638.

Graziano, M. S., and Aflalo, T. N. (2007). Rethinking cortical organization: moving away from discrete areas arranged in hierarchies. Neuroscientist 13, 138-147.

Grinvald, A., Lieke, E., Frostig, R. D., Gilbert, C. D., and Wiesel, T.N. (1986). Functional architecture of cortex revealed by optical imaging of intrinsic signals. Nature 324, 361-364.

Kaas, J. H. (1990). "Processing areas and modules in the sensory-perceptual cortex," in Signal and Sense: Local and Global Order in Perceptual Maps, eds G. M. Edelman, W. E. Gall and W. M. Cowan (New York, NY: Wiley-Liss, Inc.), 67-82.

Kaas, J. H. (1997). "Theories of visual cortex organization in primates," in Cerebral Cortex: Extrastriate Cortex 
in Primates, eds K. S. Rockland, J. H. Kaas and A. Peters (New York, NY: Plenum), 91-125.

Kaas, J. H., and Morel, A. (1993). Connections of visual areas of the upper temporal lobe of owl monkeys: the MT crescent and dorsal and ventral subdivisions of FST. J. Neurosci. 13, 534-546.

Kaskan, P.M.,Lu,H.D., Dillenburger, B.C., Kaas, J. H., and Roe, A. W. (2009). The organization of orientation-selective, luminance-change and binocularpreference domains in the second (V2) and third (V3) visual areas of New World owl monkeys as revealed by intrinsic signal optical imaging. Cereb. Cortex 19, 1394-1407.

Kaskan, P. M., Lu, H. D., Dillenburger, B. C., Roe, A. W., and Kaas, J. H. (2007). Intrinsic-signal optical imaging reveals cryptic ocular dominance columns in primary visual cortex of New World owl monkeys. Front. Neurosci. 1, 67-75.

Kim, D. S., Matsuda, Y., Ohki, K., Ajima, A., and Tanaka, S. (1999). Geometrical and topological relationships between multiple functional maps in cat primary visual cortex. Neuroreport 10, 2515-2522.

Kisvarday, Z. F., Buzas, P., and Eysel, U. T. (2001). Calculating direction maps from intrinsic signals revealed by optical imaging. Cereb. Cortex 11, 636-647.

Krubitzer, L.A., and Kaas, J.H. (1990a). Convergence of processing channels in the extrastriate cortex of monkeys. Vis. Neurosci. 5, 609-613.

Krubitzer, L. A., and Kaas, J. H. (1990b). Cortical connections of MT in four species of primates: areal, modular, and retinotopic patterns. Vis. Neurosci. 5, 165-204.

Lagae, L., Raiguel, S., and Orban, G. A. (1993). Speed and direction selectivity of macaque middle temporal neurons. J. Neurophysiol. 69, 19-39.

Lu,H.D., and Roe,A.W.(2008). Functional organization of color domains in V1 and V2 of macaque monkey revealed by optical imaging. Cereb. Cortex 18, 516-533.

Lund, J. S., Yoshioka, T., and Levitt, J. B. (1993). Comparison of intrinsic connectivity in different areas of macaque monkey cerebral cortex. Cereb. Cortex 3, 148-162.

Malach, R., Tootell, R.B., and Malonek, D. (1994). Relationship between orientation domains, cytochrome oxidase stripes, and intrinsic horizontal connections in squirrel monkey area V2. Cereb. Cortex 4, 151-165.

Maldonado, P. E., Godecke, I., Gray, C. M., and Bonhoeffer, T. (1997). Orientation selectivity in pinwheel centers in cat striate cortex. Science 276, 1551-1555.

Malonek, D., Tootell, R. B., and Grinvald, A. (1994). Optical imaging reveals the functional architecture of neurons processing shape and motion in owl monkey area MT. Proc. R. Soc. Lond., B, Biol. Sci. 258, 109-119.

Manger, P., Sum, M., Szymanski, M., Ridgway, S. H., and Krubitzer, L. (1998). Modular subdivisions of dolphin insular cortex: does evolutionary history repeat itself? J. Cogn. Neurosci. 10, 153-166.

Maunsell, J. H., and Van Essen, D. C. (1983a). Functional properties of neurons in middle temporal visual area of the macaque monkey. I. Selectivity for stimulus direction, speed, and orientation. J. Neurophysiol. 49, 1127-1147.

Maunsell, J. H., and Van Essen, D. C. (1983b). Functional properties of neurons in middle temporal visual area of the macaque monkey. II. Binocular interactions and sensitivity to binocular disparity. J. Neurophysiol. 49, 1148-1167.

Mountcastle, V. B. (1997). The columnar organization of the neocortex. Brain 120, 701-722.

Muller, J. R., Metha, A. B., Krauskopf, J., and Lennie, P. (2001). Information conveyed by onset transients in responses of striate cortical neurons. J. Neurosci. 21, 6978-6990.

Ohki, K., Chung, S., Kara, P., Hubener, M., Bonhoeffer, T., and Reid, R. C. (2006). Highly ordered arrangement of single neurons in orientation pinwheels. Nature 442, 925-928.

Op de Beeck, H. P., Haushofer, J., and Kanwisher, N. G. (2008). Interpreting fMRI data: maps, modules and dimensions. Nat. Rev. Neurosci. 9, 123-135.

Purves, D., Riddle, D. R., and LaMantia, A.S. (1992). Iterated patterns of brain circuitry (or how the cortex gets its spots). Trends Neurosci. 15, 362-368.

Raiguel, S., Van Hulle, M.M., Xiao, D.K., Marcar, V.L., and Orban, G.A. (1995). Shape and spatial distribution of receptive fields and antagonistic motion surrounds in the middle temporal area (V5) of the macaque. Eur. J. Neurosci. 7, 2064-2082.

Ramsden, B. M., Hung, C. P., and Roe, A. W. (2001). Real and illusory contour processing in area $\mathrm{V} 1$ of the primate: a cortical balancing act. Cereb. Cortex 11, 648-665.

Rasband, W. (2007). ImageJ. Bethesda, MD: US National Institutes of Health.

Rosa, M. G., and Elston, G. N. (1998). Visuotopic organisation and neuronal response selectivity for direction of motion in visual areas of the caudal temporal lobe of the marmoset monkey (Callithrix jacchus): middle temporal area, middle temporal crescent, and surrounding cortex. J. Comp. Neurol. 393, 505-527.

Rosa, M. G., and Schmid, L. M. (1995). Visual areas in the dorsal and medial extrastriate cortices of the marmoset. J. Comp. Neurol. 359, 272-299.

Shmuel, A., and Grinvald, A. (1996). Functional organization for direction of motion and its relationship to orientation maps in cat area 18. J. Neurosci. 16, 6945-6964.

Swindale, N. V. (2000). How many maps are there in visual cortex? Cereb. Cortex 10, 633-643.

Swindale, N.V., Grinvald, A., and Shmuel, A. (2003). The spatial pattern of response magnitude and selectivity for orientation and direction in cat visual cortex. Cereb. Cortex 13, 225-238.

Swindale, N. V., Shoham, D., Grinvald, A., Bonhoeffer, T., and Hubener, M. (2000). Visual cortex maps are optimized for uniform coverage. Nat. Neurosci. 3, 822-826.

Tanaka, K., Hikosaka, K., Saito, H., Yukie, M., Fukada, Y., and Iwai, E. (1986). Analysis of local and wide-field movements in the superior temporal visual areas of the macaque monkey. J. Neurosci. 6, 134-144.

Tootell, R. B.,Hamilton, S. L., and Silverman, M.S.(1985).Topography of cytochrome oxidase activity in owl monkey cortex. J. Neurosci. 5, 2786-2800.

Tootell, R. B., and Taylor, J. B. (1995) Anatomical evidence for MT and additional cortical visual areas in humans. Cereb. Cortex 5, 39-55.

Ts'o, D. Y., Roe, A. W., and Gilbert, C. D. (2001). A hierarchy of the functional organization for color, form and disparity in primate visual area V2. Vision Res. 41, 1333-1349.

Van Essen, D. C., Maunsell, J. H., and Bixby, J. L. (1981). The middle temporal visual area in the macaque: myeloarchitecture, connections, functional properties and topographic organization. J. Comp. Neurol. 199, 293-326.

Weliky, M., Bosking,W.H., and Fitzpatrick, D. (1996). A systematic map of direction preference in primary visual cortex. Nature 379, 725-728.

Wong-Riley, M. (1979). Changes in the visual system of monocularly sutured or enucleated cats demonstrable with cytochrome oxidase histochemistry. Brain Res. 171, 11-28.

Xiao, D. K., Raiguel, S., Marcar, V., and Orban, G. A. (1997). The spatial distribution of the antagonistic surround of MT/V5 neurons. Cereb. Cortex 7, 662-677.

Xu, X., Collins, C. E., Kaskan, P. M., Khaytin, I., Kaas, J.H., and Casagrande, V.A. (2004). Optical imaging of visually evoked responses in prosimian primates reveals conserved features of the middle temporal visual area. Proc. Natl. Acad. Sci. U.S.A. 101, 2566-2571.

Zeki, S. (1980). The response properties of cells in the middle temporal area (area MT) of owl monkey visual cortex. Proc. R. Soc. Lond., B, Biol. Sci. 207, 239-248.

Zeki, S. M. (1974). Functional organization of a visual area in the posterior bank of the superior temporal sulcus of the rhesus monkey. J. Physiol. 236, 549-573.

Conflict of Interest Statement: The authors declare that the research was conducted in the absence of any commercial or financial relationships that could be construed as a potential conflict of interest.

Received: 12 February 2010; paper pending published: 16 March 2010; accepted: 12 May 2010; published online: 07 July 2010.

Citation: Kaskan PM, Dillenburger BC, Lu HD, Roe AW and Kaas JH (2010) Orientation and direction-of-motion response in the middle temporal visual area (MT) of New World owl monkeys as revealed by intrinsic-signal optical imaging. Front. Neuroanat. 4:23. doi: 10.3389/ fnana.2010.00023

Copyright (C) 2010 Kaskan, Dillenburger, $\mathrm{Lu}$, Roe and Kaas. This is an open-access article subject to an exclusive license agreement between the authors and the Frontiers Research Foundation, which permits unrestricted use, distribution, and reproduction in any medium, provided the original authors and source are credited. 